\title{
Research on Attention Bias Characteristics under the Consciousness Threshold of Individuals with Social Anxiety
}

\author{
Qinyu Yang*, Yaozhong Liu \\ Jinan University, Guangzhou, China \\ Email: *yqyltlt@163.com
}

How to cite this paper: Yang, Q. Y., \& Liu, Y. Z. (2020). Research on Attention Bias Characteristics under the Consciousness Threshold of Individuals with Social Anxiety. Psychology, 11, 373-384. https://doi.org/10.4236/psych.2020.112024

Received: January 10, 2020

Accepted: February 24, 2020

Published: February 27, 2020

Copyright (๑) 2020 by author(s) and Scientific Research Publishing Inc. This work is licensed under the Creative Commons Attribution International License (CC BY 4.0).

http://creativecommons.org/licenses/by/4.0/

\begin{abstract}
In this study, the improved masking detection paradigm was adopted to form stimulus picture pairs with pictures of different emotional faces and household items, to explore the attention preference characteristics of individuals with social anxiety under the threshold of consciousness. The results showed that the threshold of consciousness, under the condition of consistency, detects the target in the reaction on the negative emotions. Face images are much smaller than to detect the target in household items to the reaction time on the images, and also appear less than to detect the target in the reaction on the positive mood face images, namely high social anxiety group compared with low social anxiety, attention to negative emotional faces that exist. The results are consistent with the alertness model in the cognitive processing theory model of social anxiety.
\end{abstract}

\section{Keywords}

Social Anxiety, Attention Bias, Consciousness Threshold

\section{Introduction}

In our daily life, we are in contact with the outside world all the time-we are in a social state all the time. However, social anxiety among college students is becoming more and more obvious. Social anxiety can cause individuals to experience anxiety, panic and other emotions in daily interpersonal communication. They are afraid of being stared at the same time as others. If not corrected or improved in a timely manner, severe social anxiety disorder may develop. As one of the most common social disorders (Bandelow \& Michaelis, 2015), social anxiety disorder is the third biggest threat to human mental health after depres- 
sion and alcohol abuse (Magee et al., 1996). Therefore, intervention and treatment before social anxiety develops into social anxiety disorder have become the focus and difficulty of research in many fields. Over the past decade, the study and treatment of psychological theory and anxiety disorders have increasingly shifted to the information processing paradigm derived from experimental cognitive psychology and social psychology to understand the cognitive basis of anxiety. The cognitive behavior model of social anxiety points out that attention bias is a key factor in the maintenance of social anxiety symptoms, and it has been proved by extensive studies (Mogg \& Bradley, 2018).

There are great differences in the models of attention preference of individuals with social anxiety, which can be divided into static attention preference and dynamic attention preference. For example, in the static attentional bias model, Clark and Well proposed the avoidance hypothesis, believing that individuals with social anxiety disorder would enter self-focused attention in social situations, thus inhibiting the processing of external cues (Clark \& Wells, 1995). Rapee and Heimberg proposed the vigilance hypothesis that individuals with social anxiety disorder would be more alert to threat cues (Rapee \& Heimberg, 1997). And the attention of the dynamic deflection model theory argues that attention bias may experience different stages of attention, for example, Mogg scholar proposed "alert - avoid" hypothesis arguing that: social anxiety disorder experience first to get into an automated initial alert, with rising anxiety, immediately into avoiding state in order to ease the anxiety (Mogg et al., 2004). Amir and Elias et al. proposed the hypothesis of attention disconnection difficulty, arguing that individuals with social anxiety disorder did not show obvious vigilance in the early stage of attention, but could not avoid it as normal individuals after attention was captured by threat cues. In other words, they could not get rid of such cues by paying attention for a long time (Amir, Elias, Klumpp, \& Przeworski, 2003).

At present, most of the researches on attention bias of social anxiety use stimulus materials such as word pairs and face pairs, while only a few scholars use non-social cue pictures such as household items in daily life as reference stimuli for research. However, when using word pairs as stimulus material, some researchers found excessive vigilance against threat stimuli (Pishyar et al., 2008), others reported patterns of excessive vigilance and attentional avoidance (Vassilopoulos, 2005), and others reported no bias (Mansell et al., 2002). Therefore, the relevance and validity of word stimuli is challenged in interpreting probe point detection tasks. Researchers have questioned the nature of threats caused by words, the ecological validity of words, or the priming effects that may result from the frequency and familiarity of specific words (Bradley et al., 1998; Mogg \& Bradley, 1999). In fact, for people with social anxiety disorder, social threats often include other people, as well as their expressions and reactions (Clark \& Wells, 1995; Rapee \& Heimberg, 1997). When people were shown pictures of emotional faces as stimulus material, the result of the probe was as difficult to reconcile as the word stimulus. Therefore, some scholars have proposed that the most convincing evidence is the use of some stimuli that are obviously repre- 
sentative in real social situations when the detection point detection task is used to study the attention bias related to social anxiety (Lowe, 2015). In these studies, avoidance of social information (pictures of faces) has been demonstrated (Chen et al., 2002; Mansell et al., 1999). In our daily life, the face is just we will observe noticed a small part of the picture, in order to be able to detect any turn our attention to or away from the face of the overall trend (whatever its valence), so choose something in the room may appear when interacting with people face represents the household items pictures to enhance the authenticity of the experiment and real sex, provide a more ecological effective measure participants to realistic social stimulation reaction method (Sears et al., 2018). If face-pair stimulus materials can help us detect emotional stimuli, face-object pair materials allow participants to freely allocate attention between faces and household items, thus helping us better analyze the strategic bias in attention distribution of socially anxious individuals affected by situational contingencies (Kret et al., 2015). At present, there are only relevant studies at the time point of $500 \mathrm{~ms}$ above the threshold of consciousness, and different processing systems are usually involved in below and above the threshold of consciousness (Kiss \& Eimer, 2008; Lazarov et al., 2016). Therefore, this paper will follow the experimental research methods and paradigms previously used by Chen et al. (2002), adopt the improved masking point detection paradigm, and use face pairs composed of emotional face pictures and household items pictures as experimental materials to explore the differences in attention bias of individuals with social anxiety under the consciousness threshold.

\section{Methods}

\subsection{The Participants}

First of all, the experiment selected 168 college students in the system of questionnaire survey, questionnaire contains social phobia scale (SPS) and social anxiety scale (SIAS), stay after participants fill out the questionnaire by using SPSS software to eliminate invalid data and select the top $9 \%$ and $9 \%$ of the subjects after the 15 respectively as high social anxiety group and low social anxiety group participants after the experiment. At the same time, in order to ensure the smooth progress of the experiment, the selected subjects should be ensured: 1) visual acuity or corrected visual acuity is above 1.0 ; 2) there is no problem of color weakness or color deficiency; 3 ) in good mental condition; 4) right-handed. Table 1 shows the demographic data of the two groups of subjects. It can be seen from the table that there is no significant difference in age between the two groups of subjects, while there is a significant difference in the total score of the questionnaire. See table 1 for detailed data.

\subsection{The Questionnaire}

At present, most foreign researchers usually use the social fear scale (SPS) and social interaction anxiety scale (SIAS) developed by Mattick et al., as tools to 
Table 1. Demographic data of high social anxiety group and low social anxiety group.

\begin{tabular}{|c|c|c|c|}
\hline & High social anxiety group & low social anxiety group & \\
\hline & $\mathrm{N}=15$ & $\mathrm{~N}=15$ & \\
\hline Gender: & male: 7 female: 8 & male: 7 female: 8 & \\
\hline Age (years) & $22.67(2.64)$ & $23.87(2.39)$ & 0.051 \\
\hline $\begin{array}{l}\text { The total score of } \\
\text { the questionnaire }\end{array}$ & $95.93(7.42)$ & $50.6(5.36)$ & $2.683^{\star * *}$ \\
\hline
\end{tabular}

measure the level of individual social anxiety. The SPS scale and SIAS scale are usually used as two subscales in a larger scale, mainly to measure the anxiety and fear emotions of the subjects in the interaction with others and in the observed social situation. Later, the two scales were translated and revised by the clinical psychology department of Peking University to make them more suitable for the research of Chinese subjects. After the revision, the internal consistency reliability of the SPS scale was 0.904 , the fractional half reliability was 0.865 , and the retest reliability was 0.849 . The internal consistency reliability of the revised SIAS scale was 0.874 , the fractional half reliability was 0.862 , and the retest reliability was 0.863 , all of which met the standard requirements of the experimental measurement.

The Beck depression questionnaire (BDI), the most widely used self-rating scale for depression, was originally constructed by Beck and colleagues (1961) to assess depression in adolescents and adults, and was revised and refined in 1979 and 1996, respectively. The second edition of BDI, revised by Beck et al in 1996, will be used in this study. This version of BDI consists of 21 items, all of which consist of scores ranging from 0 to 3 , while the revised version of BDI is also demonstrated by the adaptability of the results of Hong Kong adolescents.

The state-trait anxiety inventory (STAI) was developed by scholar Spielberger in 1977 and revised in 1983. The scale is mainly composed of two parts: the state anxiety questionnaire ( $\mathrm{s}-\mathrm{ai}$ ) and the trait anxiety questionnaire ( $\mathrm{t}-\mathrm{ai})$. Each questionnaire contains 20 items, with a total of 40 items. Among them, s-ai is mainly used to evaluate the anxiety experience of the subjects in the current or recent period, with a high volatility. T-ai was used to assess the subjects' constant anxiety experience, which was generally stable and sustained. All scales adopted the four-level scoring method of $1-4$, and relevant literatures have shown that the scale has good reliability and validity.

\subsection{The Experimental Materials}

In order to enhance the authenticity and the feasibility of the experiment, avoid emotional mixed effect of face recognition, this study decided to adopt the Chinese Academy of Sciences institute of psychological preparation of revised Chinese expression system of mood, face images as faces in the study of this material, choose happy mood face images and pictures of the anger faces all 16 as a positive mood picture materials and negative emotions, face image materials, in- 
cluding two different mood face try to choose the same or similar images, each half of the men and women. As neutral reference stimuli, the pictures of household items were screened out from the Internet by 64 pictures of the most common household items in daily life (such as clocks, sofas, vacuum cleaners and telephones) and paired with pictures of different emotional faces. At the same time, 24 neutral pictures of irrelevant materials (such as cars) were selected as the pairs of practice experimental materials before the formal experiment. All the pictures were uniformly decolorized and turned into black and white images with the same background. The picture size was $170 \times 170 \times 24 \mathrm{~b}$. The pictures were paired in pairs: household objects $(\mathrm{w}-\mathrm{w})$ to 16 pairs, positive emotional faces $(\mathrm{J})$ to household objects (W) to 16 pairs, negative emotional faces $(\mathrm{X})$ to household objects (W) to 16 pairs.

\subsection{Measurement of Attentional Bias}

The research experiment under consciousness threshold mainly adopts the improved masking technology of point detection paradigm to investigate. Formally in the whole experiment process, the first screen may render a $2 \mathrm{~cm} * 2 \mathrm{~cm}$ of the "+" $800 \mathrm{~ms}$, "+" disappears, the screen will appear any pictures to present in the form of diagonal $14 \mathrm{~ms}$, pictures to go off the screen again and experimental activate the same black and white picture size meaningless pictures covered the original experimental materials as masking stimulus presentation $186 \mathrm{~ms}$. After the masking stimulus disappeared, a detection target was randomly presented at the center of the previous image: "E" or "F". If the detection target was "E", the subject should press the "E" key with his left hand quickly and accurately. If the letter "F" appeared, he should press the letter " $F$ " key with his right hand as soon as possible. If no response is made in time, the detection point will disappear after $1500 \mathrm{~ms}$ and continue to the next trail. Experiments using 3 (image classification: positive emotion faces - household items, negative emotions faces household items, household goods, household goods) $\times 2$ (social anxiety level: high social anxiety, low social anxiety) $\times 2$ (consistency: probe point position and mood to face location, detection point location and emotional face position inconsistent) mixed design experiment. Among them, the inter-group variables are social anxiety level, the intra-group variables are picture classification and consistency, and the dependent variables are response time of detection point judgment. At the same time, in order to maximize the cost of shifting attention in response time, all stimulus pairs in the experiment were displayed diagonally on the computer screen at a 45-degree Angle (upper left and lower right corner or upper right and lower left corner). In addition, in order to solve the problem that points in the point detection task will be detected by peripheral vision, this study will use the small letter "E" or " $F$ " instead, and the subjects must indicate the existence of which letter.

After 12 practice trails, each subject was required to complete two block formal experiments, including $\mathrm{j}-\mathrm{w}$ consistent, $\mathrm{j}-\mathrm{w}$ inconsistent, $\mathrm{x}-\mathrm{w}$ consistent, $\mathrm{x}-\mathrm{w}$ inconsistent and $\mathrm{w}-\mathrm{w}$ trail 12 each. In each trail, the emotional picture, the ob- 
ject picture, the location of the detection target and the detection target " $E$ " or "F" are all random and balanced, and each pair of images in each block appears only once. After all the experiments were completed, questionnaires were asked about the subjects' status-trait anxiety and depression levels. After completing all experiments and questionnaires, the subjects will be paid a certain amount of experimental remuneration.

Among them, all the experimental programs mentioned above are programmed on the computer using e-prime. The specific experimental process is shown in Figure 1.

\section{Analysis}

The subjects included 14 boys and 16 girls. SPSS and other statistical software were used to screen out subjects with an average accuracy of more than $90 \%$ from 30 people in the experiment. At the same time, only the data with correct response were selected, and the data with less than $200 \mathrm{~ms}$ and more than $1000 \mathrm{~ms}$ were excluded, and the extreme data with plus or minus three standard deviations were excluded. In the end, 4 of the 30 subjects who did not meet the conditions were excluded, and the responses of 26 subjects were finally selected for further analysis.

According to the research method of Koster et al. (2004), we defined the attention and vigilance bias score as: under the condition of consistency, when the average response of the detection target appears on the emotional face is greater than that of the object to the response, it indicates that there is attention avoidance. When the average response of the target on the emotional face was smaller than the response of the object to the object, attention alertness was indicated. Similarly, the attention disengagement bias score is defined as: under inconsistent conditions, when the response of the detection target appears at the position of the object in the emotion-object pair is greater than that of the object pair, then the difficulty of attention disengagement occurs; When the response time of detecting the target at the position of the object in the emotion-object pair is less than the response time of the object at the position of the object in the object pair, the emergence of attention detachment facilitation is indicated. Among them, Table 2 shows the response time and standard deviation of subjects in high and low social anxiety group under five different conditions.

1) Pay attention to the alert

2 (social anxiety level: high social anxiety group, low social anxiety group) $\times 3$ (experimental conditions: $\mathrm{j}-\mathrm{w}$ consistent, $\mathrm{x}-\mathrm{w}$ consistent and $\mathrm{w}-\mathrm{w}$ consistent) repeated measure anova was performed for the high and low social anxiety

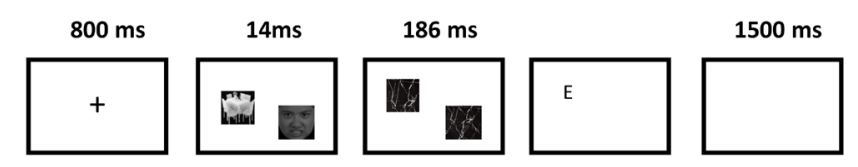

Figure 1. Flow chart of the sub-threshold point stimulus task ( $\mathrm{x}-\mathrm{w}$ image pair, example of detecting target and face image location inconsistency). 
Table 2. Responses of the high-social anxiety group and the low-social anxiety group to $\mathrm{j}-\mathrm{w}$ and $\mathrm{x}-\mathrm{w}$ images under the threshold of consciousness (Unit: $\mathrm{ms}$ ).

\begin{tabular}{cccccc}
\hline \multirow{2}{*}{$\begin{array}{c}\text { Detection points and face pictures } \\
\text { stimulate = Position consistency }\end{array}$} & \multicolumn{2}{c}{ high social anxiety group } & \multicolumn{2}{c}{ low social anxiety group } \\
\cline { 3 - 6 } & Consistent & 534.85 & 56.73 & 554.57 & 38.78 \\
\hline \multirow{2}{*}{$\mathrm{J}-\mathrm{w}$} & 525.77 & 54.03 & 553.64 & 44.81 \\
& Inconsistent & 490.23 & 23.41 & 555.00 & 47.34 \\
\multirow{2}{*}{$\mathrm{X}-\mathrm{w}$} & Consistent & 525.54 & 55.24 & 550.00 & 35.34 \\
& Inconsistency & 531.31 & 57.80 & 566.00 & 38.80 \\
\hline
\end{tabular}

Note: J-w: positive face-pictures of household items; X-w: negative face-pictures of household items; W-w: pictures of household items.

group. The results showed that the main effect of social anxiety level was significant $(\mathrm{F}=6.78, P<0.05)$, the main effect of experimental conditions was significant $(\mathrm{F}=9.01, P<0.05)$, and the interaction effect of social anxiety level $\times$ experimental conditions was significant $(\mathrm{F}=7.143, \mathrm{~F}<0.05)$, which required further simple effect analysis.

The simple effect analysis showed that there were significant differences in the responses of subjects in the high social anxiety group under three experimental conditions $(\mathrm{F}=10.00, P<0.01)$. After post hoc test, it was found that the response time of subjects in the high social anxiety group under the experimental condition of $\mathrm{x}-\mathrm{w}(490.23 \pm 23.41)$ was significantly lower than that under the experimental condition of $\mathrm{j}-\mathrm{w}(534.85 \pm 56.73)(P<0.01)$, and was also significantly lower than that under the experimental condition of $\mathrm{w}-\mathrm{w}(531.31 \pm 57.80)$ $(P<0.01)$. Under the experimental conditions of $\mathrm{x}-\mathrm{w}$, there was also a significant difference $(\mathrm{F}=22.369, P<0.01)$ in the response time of subjects in the high social anxiety group $(490.23 \pm 23.41)$ compared with those in the low social anxiety group $(555.00 \pm 47.34)(P<0.01)$.

2) Pay attention to escape difficulty

2 (social anxiety level: high social anxiety group, low social anxiety group) $\times 3$ (experimental conditions: $\mathrm{j}-\mathrm{w}$ inconsistency, $\mathrm{x}-\mathrm{w}$ inconsistency and $\mathrm{w}-\mathrm{w}$ ) repeated measure anova was performed for the two groups of subjects in the high and low social anxiety group with inconsistent $\mathrm{j}-\mathrm{w}, \mathrm{x}-\mathrm{w}$ inconsistency and $\mathrm{w}-\mathrm{w}$ response. The results showed that the main effect of social anxiety level was not significant $(\mathrm{F}=3.033, P=0.094)$, the main effect of experimental conditions was not significant $(\mathrm{F}=2.703, P=0.077)$, and the interaction of social anxiety level $\times$ experimental conditions was not significant $(\mathrm{F}=0.542, P=0.585)$.

Reaction after the experiment, the experimental keys should be completed subjects BDI and STAI questionnaire to measure its state-trait anxiety and depression levels, to determine the high and low anxiety group observed between two groups had no any difference is due to individual differences of social anxiety tendency is more common due to the negative impact of differences.

The independent sample $t$ test was used to analyze the questionnaire results, as shown in Table 3 below. 
Table 3. Difference analysis of high and low social anxiety group on state-trait anxiety scale and depression scale.

\begin{tabular}{|c|c|c|c|c|c|}
\hline & \multirow{2}{*}{\multicolumn{2}{|c|}{$\begin{array}{l}\text { High social anxiety group } \\
\qquad \mathrm{N}=15\end{array}$}} & \multirow{2}{*}{\multicolumn{2}{|c|}{$\begin{array}{l}\text { low social anxiety group } \\
\qquad \mathrm{N}=15\end{array}$}} & \multirow{3}{*}{$\mathrm{t}$} \\
\hline & & & & & \\
\hline & M & $\mathrm{SD}$ & M & SD & \\
\hline State anxiety & 41.07 & 4.75 & 30.85 & 2.85 & $6.656^{* * *}$ \\
\hline Trait anxiety & 41.77 & 3.75 & 30.62 & 2.72 & $9.462^{* * *}$ \\
\hline Depression & 10.76 & 3.54 & 3.69 & 1.11 & $6.880^{* * *}$ \\
\hline
\end{tabular}

Note: ${ }^{*}$ for $P<0.05,{ }^{* *}$ for $P<0.01,{ }^{* * *}$ for $P<0.001$.

It can be seen from Table 3 that the scores of the subjects in the high and low social anxiety groups on the state-trait anxiety scale and the depression scale were significantly different. Subjects in the high social anxiety group scored significantly higher on the state anxiety questionnaire than those in the low social anxiety group $\mathrm{t}(24)=6.656, P<0.001$. The score of the high social anxiety group on the trait anxiety questionnaire was significantly higher than that of the low social anxiety group, $\mathrm{t}(24)=9.462, P<0.001$. At the same time, the scores of the subjects in the high social anxiety group on the depression scale were significantly higher than those in the low social anxiety group, $\mathrm{t}(24)=6.880, P<$ 0.001 . The results showed that the anxiety of individuals with social anxiety was generally generalized, which was not only reflected in social situations, but also showed higher anxiety and depression in other situations, which was consistent with the previous results.

\section{Discussion}

The emotional face-object pairs were used as experimental materials to explore the experimental results under the threshold of consciousness. It was found that compared with the low social anxiety group, the high social anxiety group was more alert to the negative emotional face pictures. Specifically, under the condition of consistency, the response of the detection target on the negative emotional face pictures was significantly lower than that on the positive emotional face pictures.

The experimental data match the attentional bias of the vigilance theory. At the same time, the results of this experiment are basically consistent with the results of previous experiments using the same paradigm of neutral faces as control stimuli or words as stimuli under the threshold of consciousness. For example, Mogg et al. (2004) used word pairs as experimental materials, and found that subjects with high trait anxiety showed significantly lower response time than neutral words under the condition of negative word consistency, indicating attentional vigilance. Leventhal, a scholar, used $17 \mathrm{~ms}$ of time to study subthreshold characteristics in the study of smokers' attention bias, and found that smokers showed subthreshold attention alertness to tobacco-related stimuli (Le- 
venthal et al., 2008). These findings support the hypothesis that individuals with high social anxiety selectively allocate their attention to the spatial location of the threat surface presented under the consciousness threshold (Koster et al., 2004), consistent with the idea that anxiety-related biases operate automatically under the consciousness threshold for biologically related threatening stimuli, such as angry faces. A neat explanation for these findings is that the preconscious bias depends on the threat value of the stimulus to the individual, which in turn depends on the correlation between the stimulus and the individual's anxiety and learning experience. Thus, if the stimulus is assessed as threatening during the preattentional phase of processing, the initial selective attention is automatically directed to it. This view also seems to be consistent with a more general analysis of attentional and motivational processes that the motivation and significance of a stimulus depends on its relevance to the motor state of the body (goals, current events, degrees of deprivation, etc.), and that stimulus propensity is more likely to be allocated to highly significant stimuli. However, some scholars have obtained the phenomenon of the opposite processing mode of avoidance under the threshold of consciousness, and proposed that this phenomenon of avoidance may be related to the moderating or inhibiting effect of threat information on behaviors observed in some previous studies (Chajut \& Algom, 2003; Yiend \& Mathews, 2001). Center clues tasks such as some scholars to participants in the present mood faces to respond to immediately after the direction of the probe points. The results showed that high social anxiety was group probe points after a threat faces appear to judge used reaction significantly greater than neutral faces present after the probe point reaction, namely threatening stimulus to slow or inhibit the high anxiety individual behavioral responses. Same consciousness, therefore, under the threshold of experimental conditions there are distinct opposites as a result, the social anxiety individuals for the subliminal processing of the information of the negative emotions, there may be more complex processing mechanism, social anxiety individuals under what conditions will be triggered by threatening to stimulate to alleviate or restrain the behavior of the individual response mechanism? Can this mechanism be eliminated? These questions need to be further revealed by further experimental design and brain mechanism studies in the future. At the same time, this study also has limitations. First, we should expand the scope of screening. Due to the urgency of time, this study only selected 168 college students as the objects of the preliminary questionnaire survey. Therefore, the high social anxiety group was all non-clinical subjects, and the screening base was small in the early stage. Secondly, the face pictures selected in this study are all faces with emotion, and there is a lack of neutral face pictures as a reference. Future studies can add neutral face pictures to enrich experimental materials. Finally, this study mainly chooses the most common point detection paradigm as the experimental paradigm. Although point detection paradigm has the advantages of flexibility and convenience for observation, it still has certain defects compared with spatial cluing task and visual search task. Therefore, two other paradigms can be se- 
lected or combined to carry out experiments in the later research.

\section{Conflicts of Interest}

The author declares no conflicts of interest regarding the publication of this paper.

\section{References}

Amir, N., Elias, J., Klumpp, H., \& Przeworski, A. (2003). Attentional Bias to Threat in Social Phobia: Facilitated Processing of Threat or Difficulty Disengaging Attention from Threat? Behaviour Research and Therapy, 41, 1325-1335. https://doi.org/10.1016/S0005-7967(03)00039-1

Bandelow, B., \& Michaelis, S. (2015). Epidemiology of Anxiety Disorders in the 21st Century. Dialogues in Clinical Neuroscience, 17, 327-335.

Bradley, B. P., Mogg, K., Falla, S. J., \& Hamilton, L. R. (1998). Attentional Bias for Threatening Facial Expressions in Anxiety: Manipulation of Stimulus Duration. Cognition \& Emotion, 12, 737-753. https://doi.org/10.1080/026999398379411

Chajut, E., \& Algom, D. (2003). Selective Attention Improves under Stress: Implications for Theories of Social Cognition. Journal of Personality and Social Psychology, 85, 231-248. https://doi.org/10.1037/0022-3514.85.2.231

Chen, Y. P., Ehlers, A., Clark, D. M., \& Mansell, W. (2002). Patients with Generalized Social Phobia Direct Their Attention Away from Faces. Behavior Research and Therapy, 40, 677-687. https://doi.org/10.1016/S0005-7967(01)00086-9

Clark, D. M., \& Wells, A. (1995). A Cognitive Model of Social Phobia. In R. G. Heimberg, M. R. Liebowitz, D. A. Hope, \& F. R. Schneier (Eds.), Social Phobia: Diagnosis, As sessment, and Treatment (pp. 69-93). New York, NY: Guilford Press.

Kiss, M., \& Eimer, M. (2008). ERPs Reveal Subliminal Processing of Fearful Faces. Psychophysiology, 45, 318-326. https://doi.org/10.1111/j.1469-8986.2007.00634.x

Koster, E. H. W., Crombez, G., Verschuere, B., \& Houwer, J. D. (2004). Selective Attention to Threat in the Dot Probe Paradigm: Differentiating Vigilance and Difficulty to Disengage. Behaviour Research and Therapy, 42, 1183-1192.

https://doi.org/10.1016/j.brat.2003.08.001

Kret, M. E., Stekelenburg, J. J., De Gelder, B., \& Roelofs, K. (2015). From Face to Hand: Attentional Bias towards Expressive Hands in Social Anxiety. Biological Psychology, 122, 42-50. https://doi.org/10.1016/j.biopsycho.2015.11.016

Lazarov, A., Abend, R., \& Yair, B. H. (2016). Social Anxiety Is Related to Increased Dwell Time on Socially Threatening Faces. Journal of Affective Disorders, 193, 282-288. https://doi.org/10.1016/j.jad.2016.01.007

Leventhal, A. M., Waters, A. J., Breitmeyer, B. G. et al. (2008). Subliminal Processing of Smoking-Related and Affective Stimuli in Tobacco Addiction. Experimental and Clinical Psychopharmacology, 16, 301-312. https://doi.org/10.1037/a0012640

Lowe, P. A. (2015). The Revised Children's Manifest Anxiety Scale-Second Edition Short Form: Examination of the Psychometric Properties of a Brief Measure of General Anxiety in a Sample of Children and Adolescents. Journal of Psychoeducational Assessment, 33, 719-730. https://doi.org/10.1177/0734282915580763

Magee, W. J., Eaton, W. W., Wittchen, H.-U., McGonagle, K. A., \& Kessler, R. C. (1996). Agoraphobia, Simple Phobia, and Social Phobia in the National Comorbidity Survey. Archives of General Psychiatry, 53, 159-168. 
https://doi.org/10.1001/archpsyc.1996.01830020077009

Mansell, W., Clark, D. M., Ehlers, A., \& Chen, Y. P. (1999). Social Anxiety and Attention Away from Emotional Faces. Cognition and Emotion, 13, 673-690. https://doi.org/10.1080/026999399379032

Mansell, W., Ehlers, A., Clark, D., \& Chen, Y. P. (2002). Attention to Positive and Negative Social-Evaluative Words: Investigating the Effects of Social Anxiety, Trait Anxiety and Social Threat. Anxiety, Stress \& Coping, 15, 19-29.

Mogg, K., \& Bradley, B. P. (1999). Orienting of Attention to Threatening Facial Expressions Presented under Conditions of Restricted Awareness. Cognition \& Emotion, 13, 713-740.

Mogg, K., \& Bradley, B. P. (2018). Anxiety and Threat-Related Attention: Cognitive-Motivational Framework and Treatment. Trends in Cognitive Sciences, 22, 225-240. https://doi.org/10.1016/j.tics.2018.01.001

Mogg, K., Philippot, P., \& Bradley, B. P. (2004). Selective Attention to Angry Faces in Clinical Social Phobia. Journal of Abnormal Psychology, 113, 160-165.

https://doi.org/10.1037/0021-843X.113.1.160

Pishyar, R., Harris, L. M., \& Menzies, R. G. (2008). Responsiveness of Measures of Attentional Bias to Clinical Change in Social Phobia. Cognition \& Emotion, 22, 1209-1227. https://doi.org/10.1080/02699930701686008

Rapee, R. M., \& Heimberg, R. G. (1997). A Cognitive-Behavioral Model of Anxiety in Social Phobia. Behavior Research and Therapy, 35, 741-756.

https://doi.org/10.1016/S0005-7967(97)00022-3

Sears, C., Quigley, L., Fernandez, A., Newman, K., \& Dobson, K. (2018). The Reliability of Attentional Biases for Emotional Images Measured Using a Free-Viewing Eye-Tracking Paradigm. Behavior Research Methods, 51, 2748-2760. https://doi.org/10.3758/s13428-018-1147-z

Vassilopoulos, Ph., S. (2005). Social Anxiety and the Vigilance-Avoidance Pattern of Attentional Processing. Behavioural and Cognitive Psychotherapy, 33, 13-24. https://doi.org/10.1017/S1352465804001730

Yiend, J., \& Mathews, A. (2001). Anxiety and Attention to Threatening Pictures. The Quarterly Journal of Experimental Psychology Section A, 54, 665-681. https://doi.org/10.1080/713755991 


\section{Experimental Picture Material}

\section{Positive emotional face}
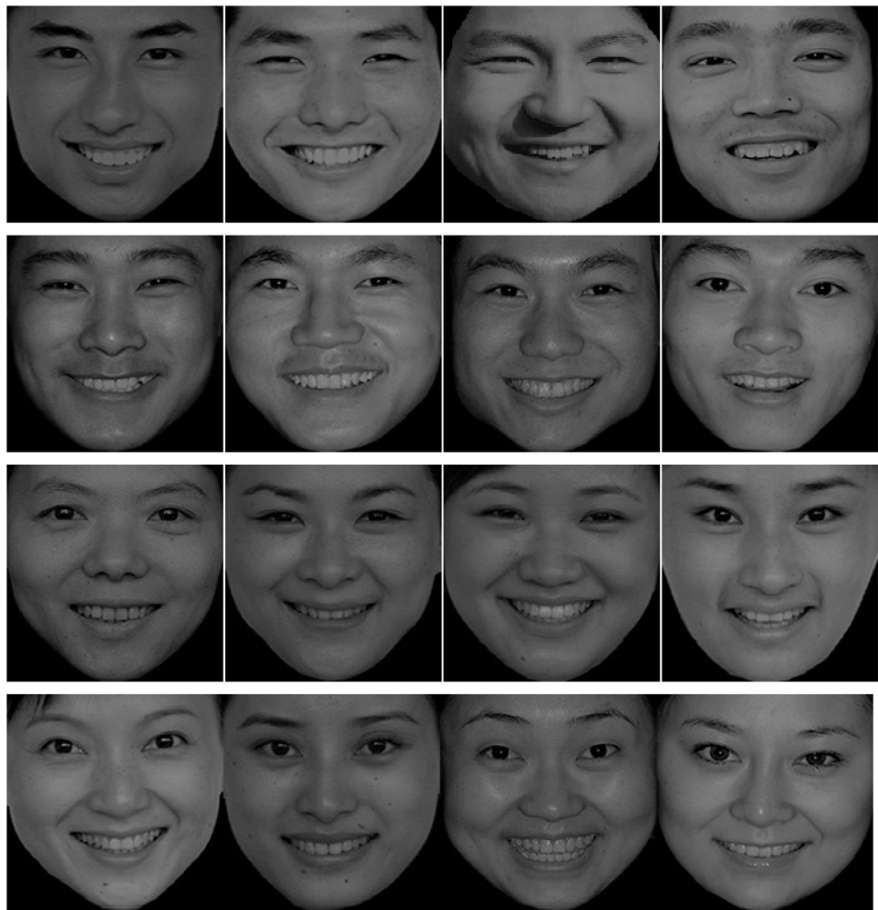

Negative emotional face
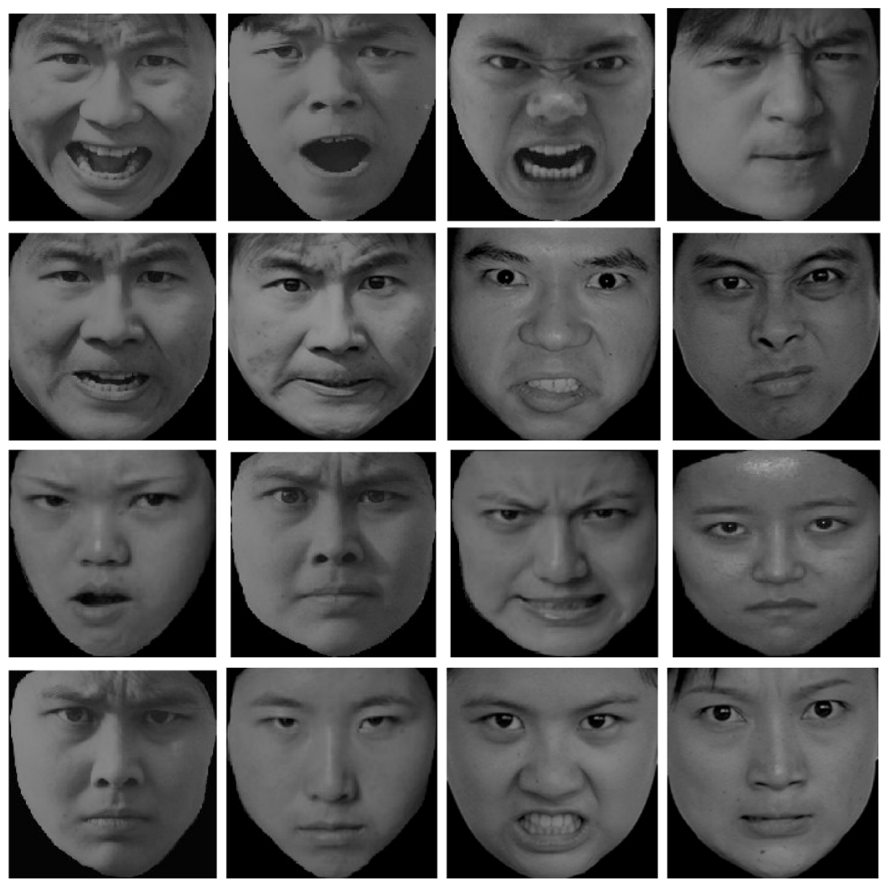